\title{
Brain research and parallel processing
}

\author{
LAWRENCE WEISKRANTZ \\ University of Oxford, Oxford, England
}

\begin{abstract}
Invited comment and counterview on "Assumptions, conceptualizations, and the search for the functions of the brain" by W. G. Webster (Physiological Psychology, 1973, 1, 346-350).
\end{abstract}

\begin{abstract}
Webster's thoughtful article is to be welcomed; the issues to which he directs attention are important for disentangling some of the constraints on the interpretation of research in physiological psychology, and we can be certain that the issues will be with us for as long as such research continues. Concern for these issues, admittedly, is always in danger of becoming an obsession, and may be akin to a priori arguments that flight in the bee is theoretically impossible, but nevertheless the logical arguments cannot be ignored.
\end{abstract}

The article touches on a number of points that provoke comment. I wish to speak briefly to just one, and then to add a gloss that may provide a somewhat different perspective. Webster makes a new and provocative suggestion when he proposes that brain manipulations (i.e., lesions, stimulation, intracerebral injections, cooling, etc.) are best understood if one assumes parallel information processing, but that they are inappropriate for the detection and study of serial processing aspects of nervous system function. He even attributes to me the view that the brain does, in fact, act as a parallel information processing device, which is a gratuitous attribution that I feel I must disclaim. He writes, "the problem with these methods [ablation and stimulation] is that while they are appropriate for the analysis and dissociation of parallel processing systems, they are simply not tuned for the detection and study of serial processing aspects of the nervous system, if there are such aspects." Moreover, he argues that the researcher is likely to be trapped by his preconceptions, because "one of the important consequences of conceptualizing the nervous system as being a parallel information processing system and then proceeding to study it with methods like ablation and stimulation is that the results will tend to support the validity of the conceptualization and will tend not to provide evidence against it."

To be influenced by one's preconceptions is a danger that is not uniquely tied to research in physiological psychology; all scientists are at risk in this regard. However, the question really turns on whether, as a matter of historical fact, inferences about serial processing have emerged from ablation and stimulation research, and if so whether there are any logical or procedural operations by which such inferences could be disconfirmed. To me it is clear that such inferences have been generated. Webster himself mentions one case-the analysis of interhemispheric integration as inferred from commissural sectioning. The maneuver is, in fact, an instructive one because the split by assumption produces parallel processing, and therefore the difference between the intact and the split preparation must be relevant to nonparallel processing, or at least to cross talk between parallel systems. A closely related example is the "disconnection syndrome" as inferred from brain damage in man (Geschwind, 1965), which certainly entails serial processing in its logical structure. The ablation work of Horel and Keating (1969) explicitly assumes a serial organization in their interpretation of the effect of a lesion that "disconnects" the amygdala from its visual input. Mishkin's (1966) work on "disconnecting" the inferotemporal region from its visual cortical input has an identical logical structure. Indeed, much of current research on the monkey's nonstriate visual cortical system is both based upon and helps bolster an interpretation cast in serial processing terms, and the hierarchical assumptions of Hubel and Wiesel's research have been appealed to explicitly in such work. [In fact, the organization that is emerging for the nonstriate visual cortex is one in which each of a number of different regions receives a direct (i.e., serial) but separate (i.e., parallel) projection from striate cortex.] An even more familiar example can be found in' any standard textbook-the serial organization and crossover in the progression from optic nerve to optic tract to 1.g.n. to optic radiations and thence to striate cortex, as inferred from the effects of lesions at different loci along the route. Quite a different example, less well developed and less secure but equally relevant, might be the work of de Molina and Hunsperger (1962) on the relationship between amygdala, hypothalamic, and midbrain focal lesions or stimulation and agonistic/defensive behavior in the cat.

The logical criteria by which the inference of serial processing can be disconfirmed have rarely been explicitly exposed by those who have made such inferences, but one self-evident maneuver is to determine whether ablations or other manipulations at different loci have equivalent behavioral effects. It should not matter in a series circuit where the disconnection occurs. 
But admittedly the real facts are not always so clear-cut (as we have seen, for example, a combination of both parallel and serial organization may obtain in the visual system), and it would make an interesting pursuit to examine the logical criteria more exhaustively. The distinction between independence and nonindependence is at best oblique to that between parallel and serial processing. One can have parallel pathways with cross talk, i.e., nonindependence (e.g., lateral inhibition), and there are several species of nonindependent relationships, e.g., convergence (multisensory cortical areas), divergence (ascending reticular formation), feedback loops (a variety of neuroendocrinological phenomena), modulation (brainstem inhibition of spinal reflexes), etc. And these two distinctions are, in turn, not simply related to that between primary and secondary effects of brain manipulations. Some secondary effects are a nuisance when one is trying to decide whether there is independence, but the analysis of "secondary" effects in their own right can give rise to interesting hypotheses about mechanisms (e.g., the residual vision following visual cortex lesions and "the two visual system" hypothesis).

Webster was kind enough to refer to some of my own pronouncements and pontifications, but he neglected to mention one that is, I think, central to the present issue. One's inferences about brain function cannot be arrived at with logical certainty, but one's confidence in their validity derives from a convergence of information from a large variety of sources-anatomical, electrophysiological, biochemical, and behavioral. The scientist is playing the role of a detective who will never secure a written confession. But neither is the evidence merely circumstantial, because he can generate testable predictions and potentially can exercise powerful control. The logical status of this convergence of information in our field has not been examined carefully as yet. I believe that it may be based on the dovetailing of two main strategies of brain research. The first deals with the brain as an "independent variable" (to use a strictly incorrect but convenient short-hand term) whereby the scientist attempts to manipulate the brain by lesions, drug injections, cooling, spreading depression, electrical stimulation, etc. The second is concerned with the brain as a "dependent variable" or "correlate," and here we examine information derived from electrophysiology, neuronal degeneration, brain concentrations, etc. It is when these logically independent streams lead to similar hypotheses and can be checked in each other's terms, via suitable bridging terms, that it would be generally agreed that progress is being achieved (Weiskrantz, 1973). Research on many systems in the brain is beginning to achieve this sort of convergence, e.g., the hypothalamus, visual cortex, amygdala, among others. Neither stream necessarily imposes an assumption or leads to an inference only of parallel processing. It is doubtless easier to arrive at a clear statement about independence of phenomena (e.g., double dissociation) than about interaction, but that as a general statement probably applies to all empirical endeavor and not just to the study of brain function.

I believe that the current inferences about brain function demonstrably extend beyond the limits of parallel processing, and are indeed rather rich in variety. No doubt one can be misled by one's assumptions and one's results. In the end, though, while it is undoubtedly important for us to be clear about what we are saying and to be aware of logical constraints and traps, it is arguably more important for us first to have something to say.

\section{REFERENCES}

de Molina, A. F., \& Hunsperger, R. W. Organization of the subcortical sy stem governing defense and flight reaction in the cat. Journal of Phy siology, 1962, 160, 200-213.

Geschwind, N. Disconnexion syndromes in animals and man. Brain, 1965, 88, 237-294 and 585-644.

Horel, J. A., \& Keating, E. G. Partial Klüver-Bucy syndrome produced by cortical disconnection. Brain Research, 1969, 16, 281-284.

Mishkin, M. Visual mechanisms beyond the striate cortex. In $R$. Russell (Ed.), Frontiers of physiological psychology. New York: Academic Press, 1966. Pp. 93-119.

Weiskrantz, L. Problems and progress in physiological psychology. British Journal of Psychcióogy, 1973, 64, 511-520. 